\title{
RILEVARE LA SECOLARIZZAZIONE: INDICATORI A GEOMETRIA VARIABILE
}

\author{
di Roberto Cartocci
}

\section{La rinnovata centralità della questione cattolica}

Il tema che intendo svolgere nelle pagine seguenti costituisce uno sviluppo - su un registro prevalentemente metodologico - di uno specifico problema cognitivo, che da alcuni anni è al centro dei miei interessi di ricerca. A tale problema, definibile con Carlo Tullio-Altan come «sindrome dell'arretratezza socioculturale italiana» $(1986 ; 1989)$, ho tentato negli ultimi anni di portare dei contributi di conoscenza empirica, approfondendo gli aspetti del comportamento elettorale, degli orientamenti cattolici e della tendenza al localismo (Cartocci 1990; 1991a; 1991b).

All'origine di tali interessi c'è la convinzione che gran parte delle gravi difficoltà che il nostro paese attraversa in questa congiuntura di transizione derivino dalla carenza di spirito pubblico e di una concezione di cittadinanza matura e responsabile; carenza che dà luogo ad un deficit di «integrazione» e ad un'ipertrofia delle pratiche «aggregative»' ${ }^{1}$, atomizzanti (in particolare due: il clientelismo e la monetizzazione del rapporto fra

Questo saggio fa parte di un più ampio programma di ricerca finanziato dal CNR (Contributo n. 9101501. CT 09). Esso costituisce una rielaborazione di una relazione presentata nella sezione di Metodologia del Convegno Nazionale dell'AIS, tenuto a Pisa dal 28 al 31 ottobre 1992. Ringrazio gli organizzatori del seminario, Maria Carmela Agodi e Luca Ricolfi, per avermi offerto questa opportunità, e inoltre Stefano Bartolini, Alessandro Bruschi, Costantino Cipolla, Piergiorgio Corbetta, Antonio De Lillo, Alberto Marradi, Franco Rositi per le loro osservazioni critiche alla prima stesura del testo. Un ringraziamento anche ad Anna De Poli dell'ADS, che mi ba fornito gli ultimi dati delle rilevazioni relative alla diffusione della stampa.

${ }^{1} \mathrm{Si}$ fa qui esplicitamente riferimento alla polarità «integrazione-aggregazione», così come definita da March e Olsen (1992), intesa dai due autori come una duplice ottica dalle molteplici implicazioni sul terreno dello studio delle organizzazioni, ma suscettibile di una lettura più ampia e feconda (Cartocci 1993).

RIVISTA ITALIANA DI SCIENZA POLITICA / a. XXIII, n. 1, aprile 1993 
cittadini e Stato attraverso l'abuso nell'emissione di titoli del debito pubblico), diverse per origine e funzionamento ma convergenti quanto a effetti disgreganti sul tessuto sociale. In altri termini, l'assenza di un'azione integratrice da parte delle istituzioni pubbliche, se da una parte produce direttamente disgregazione sociale, dall'altra non innalza alcun argine alla dinamica strutturalmente atomizzante del mercato, soprattutto nella sua declinazione nella società dei consumi (Cartocci 1993).

Nell'ambito di questo più generale problema cognitivo, si pone un'articolazione logicamente subordinata, volta ad evidenziare le profonde differenze territoriali esistenti fra Nord e Sud, non solo sul piano sociale, politico ed economico, ma anche su quello culturale.

È proprio su quest'ultima dimensione che verte questo saggio. La discussione sarà centrata, in particolare, sulle caratteristiche e sulla diffusione degli orientamenti cattolici, nell'intero paese come nelle sue articolazioni regionali. Il tema è naturalmente di estrema vastità e complessità, e non ho alcuna pretesa di affrontarne tutte le articolazioni. L'obiettivo è, più limitatamente, quello di effettuare - alla luce dei dati offerti dalle tecniche di rilevazione quantitativa più diffuse - una ricognizione della diffusione dei valori cattolici; ricognizione che appare particolarmente interessante in una congiuntura in cui la Chiesa si propone esplicitamente come strumento di integrazione sociale, con ripetuti appelli e prese di posizione da parte dei vertici della Conferenza episcopale italiana che denunciano le degenerazioni della politica e i suoi effetti disgreganti, come la socializzazione all'illegalità (e all'indifferenza per l'illegalità diffusa), prodotta dall'inefficienza delle istituzioni, e il ruolo svolto dalla mediazione politica nel perpetuare il sottosviluppo del Mezzogiorno $^{2}$. A queste prese di posizione ufficiali corrisponde, sul piano simbolico, il rilievo assunto negli ultimi mesi dagli arcivescovi di Milano e di Palermo in relazione allo scandalo delle tangenti e alle stragi compiute dalla mafia. In entrambi i casi i due porporati sono diventati il punto di riferimento morale an-

${ }^{2}$ Fra le prese di posizione più rilevanti sono da ricordare, in sequenza temporale, la Nota pastorale su La formazione all'impegno sociale e politico del $1^{\circ}$ maggio 1989 , il documento Chiesa italiana e Mezzogiorno: sviluppo nella solidarietà, del 18 ottobre 1989, la Nota pastorale Educare alla legalità del 4 ottobre 1991 e l'Appello alla speranza $e$ alla solidarietà del 30 giugno 1992. Sulla stessa linea è da porre poi il sostegno offerto dai rappresentanti della Chiesa all'opzione abrogazionista in occasione del referendum del 9 giugno 1991 sulla preferenza unica. 
che per settori di opinione pubblica esterni alla pur ampia platea dei cattolici praticanti ${ }^{3}$.

D'altra parte il riproporsi della questione cattolica ha rinnovato il dibattito sull'opportunità di un partito di o dei cattolici. In seguito al terremoto elettorale del biennio 1990-1992 si colgono nitidamente i segni di una ridefinizione del sistema partitico italiano e si torna a interrogarsi sulle forme e sul senso dell'unità politica dei cattolici. Se alla vigilia delle elezioni del 5 aprile il cardinale Ruini, presidente della Cei, con accenti duri e perentori aveva invitato i cattolici a votare per la Dc, nei primi giorni di ottobre alcuni intellettuali cattolici, tra cui Leopoldo Elia, Maria Eletta Martini e Alberto Monticone, davano vita al movimento «Carta 93» con lo scopo di contribuire al rinnovamento della Dc attraverso una riscoperta delle sue radici nel mondo cattolico. Una settimana dopo Mario Segni riuniva i suoi «Popolari per le riforme» in un'affollata ed entusiasta convention al Palazzo dello sport di Roma ${ }^{5}$.

$\mathrm{E}$ appunto di fronte a questo rinnovato attivismo dei vertici, istituzionali e politici, del mondo cattolico italiano che si pone l'esigenza di rilevare in primo luogo la diffusione, a livello di massa, degli orientamenti cattolici e, in secondo luogo, la loro differente distribuzione geografica. In particolare si tratta di accertare, a distanza di dieci anni, se e quanto è cambiata la geografia della secolarizzazione nel nostro paese, che avevo già fatto oggetto di una precedente ricerca, riferita al 1981 (Cartocci 1990 , cap. VI).

Questo obiettivo cognitivo solleva alcune importanti questioni metodologiche, attinenti in particolare alla scelta degli indicatori e delle unità d'analisi da utilizzare; questioni tanto più rilevanti alla luce di alcune recenti indagini effettuate mediante il ricorso a dati di sondaggio e rivolte appunto ad accertare la diffusione della fede cattolica in Italia (Garelli 1991; Brunetta e Longo 1991; Gubert 1992). Nelle prossime due sezioni sarà te-

${ }^{3}$ Una prova di questo rilievo è l'ampia eco suscitata dalla lettera pastorale del cardinale Martini, Sto alla porta (Centro Ambrosiano, Milano, 1992). Questo attivismo della gerarchia ha dato anche lo spunto per interventi critici da parte di intellettuali laici; cfr. in particolare l'articolo di Gianni Vattimo, I vescovi nuovi intellettuali, apparso su «La Stampa» del 15 novembre 1992, e il dibattito che ne è seguito su varie testate, in particolare la risposta di Rocco Buttiglione su «ll Tempo» del 21 novembre 1992.

${ }^{4}$ Il riferimento esplicito è alle considerazioni di Parisi in merito al rapporto fra l'elettorato cattolico e la Dc. Cfr. Parisi (1979, 91 ss.).

5 A ulteriore testimonianza dell'effervescenza del mondo politico cattolico, il 12 ottobre la Dc ha eletto per acclamazione Martinazzoli alla segreteria del partito. 
matizzata la problematicità del nesso fra concetti e indicatori. Cercherò poi di evidenziare alcuni limiti di cui soffrono i dati di sondaggio proprio per un'inadeguata riflessione su questo nesso in vista dell'accertamento della diffusione dei valori cattolici. Saranno poi esposti i vantaggi derivanti dal ricorso a dati aggregati invece che a dati individuali. Le sezioni finali del saggio illustreranno - ricorrendo appunto a dati aggregati - $\mathrm{i}$ cambiamenti intervenuti negli ultimi dieci anni nel processo di secolarizzazione e la diversa articolazione territoriale di questo processo, cambiamenti tali da richiedere una drastica revisione della variabile deputata a rilevare la diffusione dei valori cattolici.

\section{Scala di astrazione e indicatori}

Il punto critico da mettere a fuoco inizialmente è quello relativo alla trasformazione dei concetti in variabili. Sartori affronta il problema partendo dal triangolo di Ogden e Richards (1946), ai cui vertici troviamo il termine, il significato e il referente (fig. 1).

Il rapporto fra termine e significato viene risolto mediante una definizione «dichiarativa», che mira a ridurre i rischi di ambiguità e di equivocità . Il rapporto fra referente e significato

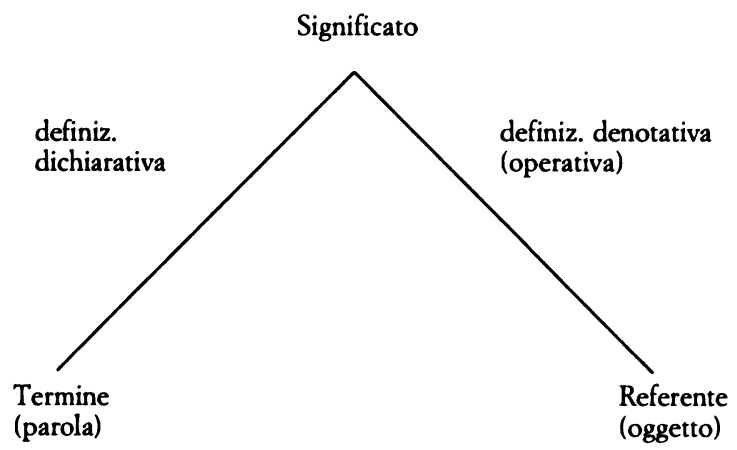

FIG. 1. Il triangolo di Ogden e Richards nella formulazione di Sartori

6 Per ambiguità si intende la scarsa chiarezza della relazione termine-significato dovuta alla presenza di omonimie e sinonimie (Sartori 1984, 72). 
viene risolto dalla definizione denotativa, il cui compito è ridurre la vaghezza della relazione fra termini e referenti ${ }^{7}$. Una sottospecie di definizione denotativa, la definizione operativa, consente la rilevazione empirica dei concetti, processo che comporta comunque un impoverimento e una certa dose di distorsione della connotazione, o intensione (1984, 22-31).

Sartori ha avuto anche il merito di richiamare l'attenzione degli scienziati sociali sull'importanza della scala di astrazione, che consente di organizzare i concetti secondo criteri di crescente connotazione - o di decrescente denotazione, che è lo stesso (Sartori 1984, 44 ss.) ${ }^{8}$. In altri termini, la scala di astrazione (o di generalità) permette di passare da concetti con ampia denotazione (e ridotta connotazione) a concetti più specifici, dotati di un più ricco numero di attributi ma di un'estensione più ridotta.

Una limpida illustrazione della scala di astrazione è fornita dalla geometria euclidea. Ad un elevato livello di generalità troviamo il concetto di «poligono»; a livelli di generalità inferiori troviamo, nell'ordine, $\mathrm{i}$ concetti di «quadrilatero», «parallelogrammo», «rettangolo», «quadrato». Ogni concetto è dotato di una definizione dichiarativa precisa. Ad esempio, definito il concetto di parallelogrammo facendo riferimento ad alcune caratteristiche (quattro lati, a due a due paralleli), il concetto di rettangolo si definisce arricchendo la connotazione di un ulteriore attributo (quattro angoli uguali). Per definire il concetto di quadrato occorre aggiungere alle precedenti caratteristiche anche quella dei lati uguali (fig. 2)

Nell'architettura deduttiva della geometria euclidea ogni concetto è definito in modo non ambiguo; così pure non esistono margini di incertezza per identificarne i referenti. In questo ambito troviamo tre tipi di connessioni univoche o, secondo la terminologia introdotta da Marradi (1991; 1993), altrettanti tipi di «giunti rigidi»: 1) il giunto che unisce il termine al significato (segmento di sinistra nella fig. 1); 2) il giunto che unisce il si-

7 La vaghezza si riferisce all'impossibilità di decidere se alcuni referenti fanno o meno parte dell'estensione di un concetto.

8 Per ulteriori trattazioni cfr. Marradi $(1984 a, 14$ ss.), Bruschi $(1990,46)$ e Fisichella $(1985,61)$.

9 D'altra parte resta vero quanto evidenziato da Marradi (1984a, 15), e cioè che un concetto è situato su una pluralità di scale di astrazione. Ad esempio, la scala riportata nella fig. 2 può essere modificata sostituendo al concetto di rettangolo quello di rombo, che è posto allo stesso livello di generalità, anche se è connotato in maniera diversa (un parallelogrammo con quattro lati uguali). 
+ estensione

+ intensione

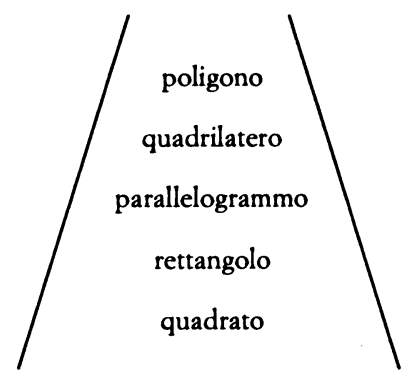

FIG. 2. Un esempio di scala di astrazione nell'ambito della geometria euclidea

gnificato ai referenti (segmento di destra nella fig. 1); 3 ) il giunto che unisce ogni concetto a tutti gli altri nella scala di astrazione; in altri termini il rettangolo (o il rombo) non possono che essere situati fra il parallelogrammo e il quadrato.

Questa rigidità dei giunti, dovuta al carattere deduttivo della geometria euclidea, le ha consentito di attraversare indenne $\mathrm{i}$ secoli e i continenti. Essa può essere messa in discussione solo modificandone alcune premesse logiche, come ad esempio hanno fatto Lobacevskij e Riemann ridefinendo il quinto postulato di Euclide.

Questo ampio riferimento all'esempio tratto dalla geometria euclidea mi permette di istituire un adeguato termine di confronto con quanto accade nella pratica di ricerca empirica del sociologo, del politologo e dello psicologo sociale. Per costoro la scala di astrazione consente di operativizzare una proprietà ad alto livello di generalità mediante il ricorso ai suoi indicatori, cioè a proprietà dotate di tre caratteristiche:

a) maggiore intensione (e minore estensione) rispetto alla prima;

b) connessione di natura semantica con la proprietà complessa;

c) possibilità di essere rilevate empiricamente ${ }^{10}$.

$\mathrm{Dal}$ punto a) deriva la conseguenza che il ricorso ad un indicatore consente di rilevare solo un aspetto della proprietà complessa; da cui la necessità, già sottolineata da Lazarsfeld (1967, 190), di ricorrere ad una pluralità di indicatori per rilevare la stessa proprietà.

Dai punti b) e c), congiuntamente considerati, deriva una

${ }^{10}$ Desumo integralmente questa caratterizzazione degli indicatori dalla trattazione fattane da Marradi (1984a, 32-42). 
conseguenza a mio parere più rilevante, in quanto consente di caratterizzare meglio le specificità epistemologiche della ricerca empirica nelle scienze sociali. Il termine chiave è semantica. La semantica «ha per oggetto lo studio dei significati delle espressioni che compongono il linguaggio» (Bruschi 1990, 44), dunque consiste nello studio del giunto fra termini e significati (segmento di sinistra della fig. 1). Ma fuori dal dominio dei linguaggi assiomatici, quando cioè inizia la ricerca empirica, allora la semantica si riferisce ai giunti fra termini e significati nel linguaggio ordinario, nel linguaggio tecnico dello scienziato sociale e nel passaggio dall'uno all'altro, in un saliscendi su scale di astrazione assai meno levigate di quella che discende dal concetto di «poligono».

Ciò implica la perdita di quella univocità delle connessioni rilevata nell'ambito della geometria euclidea. Nella ricerca empirica la connessione semantica fra proprietà non direttamente rilevabili e indicatori non è data una volta per tutte, ma deve essere sempre accertata ex novo al variare dei contesti, dei tempi, degli assetti culturali e delle unità d'analisi; da cui la natura «stipulativa» del rapporto di indicazione (Marradi 1984, 34). Nella ricerca empirica (ma, ancora prima, nella vita quotidiana) la storia e la geografia sottopongono la connessione semantica fra $\mathrm{i}$ termini e $\mathrm{i}$ rispettivi significati ad una tensione continua: una connessione individuata nel contesto $X$ al tempo $t$ ' può non sussistere nel contesto $\mathrm{Y}$ e/o al tempo $t^{\prime \prime}$. E questa tensione si riflette direttamente sui referenti empirici, così come sull'area di sovrapposizione semantica fra più concetti, dando vita ad assetti a geometria variabile.

In sintesi, nella ricerca empirica $i$ giunti individuati in precedenza (nel triangolo termine-significato-referente e tra gli scalini della scala di astrazione) sono, e non possono che essere, «giunti elastici», sottoposti all'usura della vita quotidiana e alla diversità dei contesti. Usura che peraltro non può che accrescersi in un periodo di accentuato mutamento sociale e politico, economico e culturale, che influisce sul ricambio del patrimonio concettuale, sia per quanto riguarda i concetti «tecnici» delle scienze sociali, sia per quanto riguarda i concetti della vita quotidiana, che ai primi vengono connessi mediante il rapporto di indicazione ${ }^{11}$.

\footnotetext{
$"$ Si pensi ad esempio all'usura cui sono andati soggetti due tipici indicatori di partecipazione politica: l'iscrizione a un partito o a un sindacato. Il presupposto seman-
} 


\section{Legami semantici e sintattici}

La rilevazione empirica di una proprietà non direttamente rilevabile passa dunque attraverso le seguenti fasi:

1) individuazione di una pluralità di indicatori;

2) loro trasformazione in variabili mediante la definizione operativa;

3) costruzione di un indice che sintetizzi l'informazione ottenuta attraverso la pluralità di variabili.

Alla luce di una feconda distinzione individuata da Ricolfi (1992), è possibile ricondurre le prime due fasi all'interpretazione «semantica» del rapporto di indicazione; la terza è invece riconducibile a quella «sintattica». L'interpretazione semantica privilegia la dimensione verticale, ovvero il saliscendi lungo la scala di astrazione, e si sviluppa attraverso le considerazioni che individuano una connessione di significato tra proprietà di estensione/intensione diversa. L'altra interpretazione si concentra sulla dimensione orizzontale, ovvero sulle relazioni (sotto forma di covariazioni) tra variabili.

Sono ad esempio considerazioni semantiche quelle che tengono conto del diverso rapporto fra concetti e indicatori al variare del tipo di unità, anche a parità di contesto. Si pensi alla proprietà "possesso dell'abitazione in cui si abita». Se le unità della ricerca sono gli individui, il possesso dell'abitazione è un indicatore di reddito: in generale (e con ampie eccezioni), chi è padrone della propria casa è più ricco di chi paga l'affitto. Se le unità sono aggregati territoriali (comuni e province), la quota di persone che abitano un'abitazione di loro proprietà è indicatore di tutt'altro concetto: quello di marginalità territoriale. Infatti questa quota è più elevata nelle zone di esodo (come le zone interne e montane, dove i pochi che restano abitano nelle case di proprietà, e dove comunque i costi degli immobili sono più contenuti) e più bassa nelle zone di immigrazione delle giovani coppie (tipico il caso delle cinture delle grandi città) ${ }^{12}$.

tico che lega indicatori e proprietà generale si fonda su un assunto, spesso implicito, secondo cui l'iscrizione a un partito o a un sindacato è motivata da orientamenti ideologici, coscienza di classe, ecc., di natura universalista. È assai dubbio che questo assunto abbia ancora validità nell'Italia degli anni novanta, in cui per più di un partito si pone il dilemma dell' «azzeramento delle tessere», e in cui la maggior parte degli iscritti ai sindacati confederali è costituita da pensionati, spesso arruolati per il tramite degli enti di patronato.

${ }_{12}$ Traggo l'esempio da un'indagine pubblicata su «Il sole-24 ore» del 10 ottobre 
Prospettiva semantica

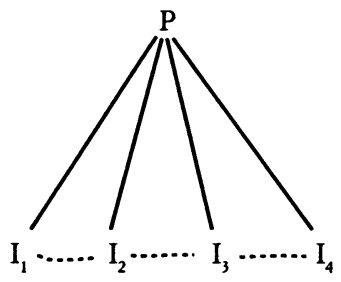

criterio: sovrapposizioni semantiche

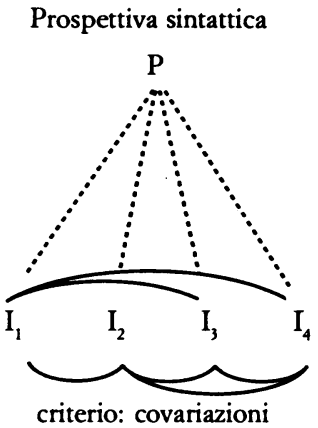

FIG. 3. Rappresentazione schematica delle due prospettive attraverso cui è possibile analizzare la relazione fra proprietà generale $(P)$ e indicatori $(I)$

Sono invece considerazioni sintattiche quelle che si concentrano sul problema di sintetizzare in un indice le informazioni fornite da una pluralità di indicatori. La fig. 3 illustra schematicamente la differenza fra le due ottiche.

In linea di principio, tra $\mathrm{i}$ due versanti le differenze sono profonde: sul versante semantico gli strumenti principali sono l'interpretazione e la retorica. Il ricercatore, quando non si limita a riproporre meccanicamente scelte operate da altri, giunge a «stipulare» una relazione fra una proprietà non direttamente rilevabile e un indicatore attraverso un'operazione di natura interpretativa, effettuata richiamando particolari aspetti dei concetti rilevanti, le specificità delle unità d'analisi utilizzate e dei contesti in cui la ricerca viene effettuata. È poi attraverso un'argomentazione che il ricercatore motiva le sue scelte in tema di indicatori di fronte alla comunità scientifica; l'obiettivo della sua esposizione è persuadere, non dimostrare more geometri$c o^{13}$. Niente di più distante dallo strumento principe del versante sintattico. Per esplorare le relazioni fra una pluralità di indicatori è fondamentale il ricorso alle tecniche statistiche multivariate, elaborate al computer ${ }^{14}$.

1988 , relativa alla qualità della vita nelle 95 province italiane. Ancora più sottile l'osservazione di Marradi, secondo cui il rapporto di indicazione può cambiare anche passando dal livello di aggregazione comunale a quello provinciale (1984a, 35).

${ }^{13}$ Sul ruolo dell'argomentazione nella giustificazione della scelta degli indicatori mi permetto di rinviare a Cartocci (1984); sulle caratteristiche strutturali dell'argomentazione rimando alle opere di Perelman (1981), Perelman e Olbrechts-Tyteca (1966) e Toulmin (1975).

${ }_{14}$ Meno rilevante un'altra differenza fra i due versanti. Quello sintattico non può attuarsi che dopo la raccolta dei dati, mentre quello semantico viene di regola percorso 
Nella pratica di ricerca, considerazioni semantiche e operazioni sintattiche sono strettamente connesse. Ciò non toglie però che oggi esistano potenti incentivi a privilegiare il versante sintattico, per almeno due ragioni.

La prima è dovuta agli effetti prodotti dalla rivoluzione tecnologica in atto, e che nell'ultimo decennio ha assunto ritmi forsennati. Grazie ad essa si sono abbattute tutte e tre le soglie che - fino a venti anni fa - si frapponevano alla diffusione di un'approfondita analisi dei dati. Queste tre soglie erano costituite rispettivamente dalla competenza tecnica specifica (conoscenza di un linguaggio di programmazione molto «vicino alla macchina»), dai costi monetari di accesso (la CPU del calcolatore centrale e il tempo-uomo dell'esperto informatico), dai cost $i$ pratici di accesso a quelle che Schadee ha chiamato «cattedrali nel deserto» (1990). Ancora nei primi anni ottanta non c'era più di un terminale per facoltà, con orari di apertura regolati da criteri burocratici e sindacali.

Oggi queste soglie non esistono più. La disponibilità di personal computer potenti e a buon mercato e di sofisticato software a costo ragionevole costituisce un potente incentivo a effettuare analisi multivariate assai complesse anche in mancanza di una piena padronanza delle loro implicazioni. La rivoluzione informatica ha spalancato sterminate praterie per gli apprendisti stregoni a caccia di scorciatoie sintattiche che colleghino ampie batterie di indicatori a concetti mal definiti ${ }^{15}$. Con questo naturalmente non voglio difendere una posizione contraria al progresso tecnico; intendo solo richiamare l'attenzione sull'accresciuta necessità di una riflessione concettuale e semantica, la cui importanza rischia di essere obnubilata dallo sfavillìo di analisi multivariate complesse, rese possibili dall'aumento delle capacità di calcolo delle macchine e dall'ampliamento della platea degli utenti ${ }^{16}$.

prima della raccolta dei dati. Tuttavia, nell'analisi secondaria, considerazioni di natura semantica sul rapporto di indicazione sono successive alla raccolta dei dati. Anzi, l'accresciuta disponibilità di dati (su cui tornerò tra breve), che caratterizza gli ultimi anni e che è destinata ad aumentare in futuro, è una potente molla all'esercizio di considerazioni semantiche ex post.

15 Come è stato recentemente osservato, ciò avviene «soprattutto quando i comandi di elaborazione sono troppo semplificati, come per esempio nel caso dell'analisi fattoriale di una delle librerie di programmi più diffuse, dove per la sua effettuazione basta digitare l'istruzione factor seguita dall'elenco delle variabili da includere nell'analisi, lasciando al computer la responsabilità di decisioni assolutamente determinanti agli effetti del risultato finale» (Corbetta 1992, 11).

${ }^{16}$ L'allargamento del ventaglio delle tecniche multivariate disponibili ha messo 
D'altronde l'esplorazione dei legami sintattici viene alimentata anche da un altro elemento strutturale che contraddistingue questo periodo rispetto a quelli immediatamente precedenti. Alludo all'accresciuta disponibilità di dati provenienti da varie fonti, pubbliche e private. Si pensi alle banche-dati interrogabili agevolmente a distanza via modem, alle varie indagini periodiche promosse da considerazioni market oriented ${ }^{17} \mathrm{e}$ alla progressiva diffusione dei sondaggi telefonici, che hanno abbattuto i costi elevati delle survey tradizionali, condotte con interviste faccia a faccia.

A fronte di questi potenti incentivi a percorrere il versante sintattico, chi scrive è del parere che si debba invece valorizzare il versante semantico. Come intendo mettere in luce nelle sezioni seguenti, una scarsa attenzione ai legami semantici fra proprietà generali e indicatori è all'origine di alcuni limiti comuni alle varie ricerche che negli ultimi anni hanno inteso accertare la diffusione dei valori cattolici in Italia.

\section{Secolarizzazione e pratica religiosa}

Anche quando si intenda rilevare il livello di secolarizzazione del nostro paese e delle sue diverse zone è imprescindible un supplemento di riflessione sulle connessioni fra termine, significato e referenti. Il termine «secolarizzazione» costituisce l'origine di una molteplicità di giunti elastici, che obbligano il ricercatore ad una chiarificazione semantica, che faccia luce nella selva delle accezioni ${ }^{18}$. Per non ripetere quanto affermato altrove (1990, cap. VI), mi limiterò a ricordare tre possibili accę-

però a disposizione anche procedure che disciplinano la scelta delle variabili subordinandole a specifici vincoli e percorsi causali, e che costringono perciò il ricercatore ad un'attenta riflessione sul loro significato. Alludo ai «modelli di equazioni strutturali», e in particolare ai «modelli di misurazione», per i quali rimando all'esaustivo manuale di Corbetta (1992, spec. 174-179) e a Giampaglia (1992).

${ }_{17} \mathrm{Mi}$ riferisco in particolare alle indagini periodiche sulla diffusione della stampa (ADS, Ispi, Isegi), ripetute con cadenze ravvicinate per controllare l'efficacia degli investimenti pubblicitari. Rispetto a molte fonti di dati istituzionali, quelle market-oriented hanno il vantaggio della tempestività. Il caso-limite è costituito dall'Auditel, le cui rilevazioni degli ascolti televisivi sono disponibili dopo poche ore a chiunque consulti il Televideo.

${ }^{18} \mathrm{Sul}$ concetto di secolarizzazione e sul ruolo della religione nella società contemporanea la letteratura è sterminata. Fra i contributi più recenti cfr. Guizzardi (1979), Wilson (1986), Berger (1987), Crespi (1988), Acquaviva e Stella (1989). 
zioni del termine, che rimandano a concetti posti a livelli diversi sulla scala di astrazione:

1) al livello più alto di generalità la secolarizzazione indica, weberianamente, il progressivo disincantamento del mondo (Entzauberung der Welt) (Weber 1919; trad. it. 1973, 41);

2) ad un livello intermedio, e in termini fenomenologici, la secolarizzazione rimanda ad una frammentazione del cosmo sacro, cioè ad una pluralizzazione dei riferimenti simbolici e a una privatizzazione dell'esperienza religiosa (Berger 1984, 147 ss.);

3) al livello di generalità più basso il termine «secolarizzazione» designa la contrazione dell'influenza sociale, culturale e politica delle istituzioni ecclesiastiche (Parisi 1978).

E quest'ultimo il livello che rende più agevole la rilevazione empirica, oltre ad essere il più rilevante ai fini di un'esplorazione dei riflessi degli orientamenti religiosi sul comportamento elettorale. Si pone tuttavia a questo punto un problema di natura squisitamente semantica: individuare gli indicatori più adeguati per rilevare i confini del mondo cattolico. Come ho già argomentato altrove (1990, 162 ss.), motivi sociologici e antropologici convergono nell'individuare nella partecipazione ai riti I'elemento centrale dell'esperienza religiosa: nel rito si attua quella connessione tra il mondo terreno e il trascendente (Geertz 1973, 112; Cazeneuve 1974, 272 ss.). Nel rito, cioè «attraverso le pratiche simboliche di riscatto della presenza» (Tullio-Altan 1992, 74), sono circoscritte in particolare quelle forme di rassicurazione che costituiscono uno degli aspetti culturalmente più rilevanti dell'esperienza religiosa ${ }^{19}$.

Dunque, al fine di rilevare l'appartenenza effettiva al mondo cattolico, gli indicatori più rilevanti sono senza dubbio quelli relativi alla pratica religiosa, in particolare la frequenza alla messa, «per il motivo che costituisce il più semplice e ricorrente momento in cui la religione cattolica è istituzionalizzata in quanto religione e non quale istituzione avente altri contenuti» (Guizzardi 1991, 293).

19 Questo elemento di rassicurazione caratterizza peraltro ogni tipo di rito, anche fuori dall'esperienza religiosa: «le celebrazioni rituali hanno ovunque essenzialmente lo stesso scopo, che è quello di garantire la sicurezza psicologica degli esseri viventi in un gruppo umano, per quanto riguarda il loro esistere individuale e collettivo, reintegrando l'ordine psico-sociale minacciato» (Tullio-Altan 1992, 137). Come ricorda ancora Tullio-Altan, «la stessa etimologia del termine "rito" ci rimanda all'etimo sanscrito "rta", che significa il principio divino dell'ordine nella natura e nella società». 
I dati di sondaggio documentano la caduta della pratica religiosa avvenuta in questo dopoguerra (Guidorossi 1984; Ricolfi 1988; Garelli 1991). Tuttavia anche queste informazioni soffrono di alcuni limiti, che da tempo sono stati esposti da Parisi:

Oltre al rispetto delle norme rituali [la frequenza alla messa] è capace di misurare il grado di interazione col gruppo sociale dei cattolici e il grado di esposizione ai messaggi culturali che lo attraversano, a cominciare soprattutto da quelli provenienti dalla istituzione ecclesiastica e dal clero. Va inoltre osservato che quando la domanda diretta a rilevarlo, che è formulata (purtroppo dal punto di vista della comparabilità) in modo diverso, assume (come prevalentemente accade) la forma «con quale frequenza lei va alla messa?» l'indicatore tende a rappresentare anche l'autoidentificazione. L'intervistato risponde infatti ad essa non come se lo si interrogasse sulla semplice frequenza di un comportamento, ma come se gli si chiedesse se è disposto a identificarsi come un buon cattolico (= credente e praticante). In questo senso è probabile che si autoclassifichino fra i praticanti regolari persone che di fatto praticano irregolarmente (Parisi 1979, 93-94).

Le considerazioni di Parisi, se confermano la centralità della dimensione rituale, mettono in luce come le risposte alla domanda sulla frequenza alla messa possono essere motivate anche dalla volontà degli intervistati di offrire una particolare immagine di sé, in linea con alcuni canoni di (presunta) desiderabilità sociale. Certamente la volontà di identificarsi - agli occhi propri e dell'intervistatore - «come un buon cattolico» attesta comunque l'adesione, più o meno sincera, ad un modello. Resta il fatto però che l'indicatore tende a sovrastimare il numero dei praticanti regolari. Anche recentemente, commentando l'esiguo numero di praticanti regolari rilevato attraverso un sondaggio, è stato osservato che «il dato ci sembra anche sovradímensionato rispetto alla frequenza reale» (Brunetta e Longo 1991, 46). Da un altro sondaggio effettuato nel 1991 «risulta che il 35\% dei cattolici "praticanti" non è andato a messa la domenica precedente all'intervista» (Nesti 1992, 50).

A parte questi limiti, intrinseci al ricorso ai dati di sondaggio $^{20}$, le più recenti ricerche (cfr. Sani 1991) convergono nell'in-

${ }^{20}$ Sui limiti dei sondaggi su questa specifica tematica si vedano, recentemente, gli interventi di Guizzardi e Margiotta-Broglio nel volume curato da Brunetta e Longo (1991a). Rilievi che concordano sostanzialmente con l'opinione recentemente espressa da Rositi, in merito alla difficoltà con cui variabili «direttamente culturali» (cioè domande e scale di atteggiamento inserite in questionari) riescono ad accertare gli effettivi orientamenti degli interessati (Rositi 1992, 5). 
TAB. 1. Frequenza ai riti religiosi in tre ricerche recenti, in percentuale

\begin{tabular}{lccc}
\hline Categorie di risposta & $\begin{array}{c}\text { Fond. Agnelli } \\
\text { anno } 1987 \\
2423 \text { casi }\end{array}$ & $\begin{array}{c}\text { ISPES } \\
\text { anno 1991 } \\
2000 \text { casi }\end{array}$ & $\begin{array}{c}\text { EVSSG } \\
\text { anno 1991 } \\
2017 \text { casi }\end{array}$ \\
\hline non risponde & - & 13,8 & - \\
mai o quasi mai & 23,2 & - & 15,9 \\
meno di una volta l'anno & - & - & 4,2 \\
una volta l'anno & - & - & 4,3 \\
qualche volta l'anno & 29,3 & 40,7 & - \\
A Natale e Pasqua/altre feste & - & - & 21,9 \\
una volta al mese & - & - & 13,2 \\
1-2 volte al mese & - & - & - \\
alcune volte al mese & 17,8 & 24,4 & - \\
una volta la settimana & - & - & - \\
tutte le settimane o più & 29,8 & 5,9 & 9,5 \\
più di una volta la settimana & - & 29,3 & 39,8 \\
totale praticanti regolari & 29,8 & & \\
\hline
\end{tabular}

Formulazione dei quesiti: Fondazione Agnelli «Con quale frequenza lei assiste ai riti religiosi?» (Garelli 1991, 59); ISPES «Se va in chiesa, con quale frequenza lo fa?» (Brunetta e Longo 1991, 46); EVSSG «A parte alcuni riti religiosi come i matrimoni, i funerali e i battesimi, ogni quanto frequenta le funzioni religiose attualmente?» (Gubert 1992, 593).

dicare che l'osservanza cattolica caratterizza oggi una netta minoranza della popolazione italiana, quantificabile intorno al $30 \%$ - come illustra la tab. 1 , in cui sono state riportate le risposte alle domande che (con diverse formulazioni) sono state utilizzate per rilevare la frequenza in chiesa nelle tre più recenti ricerche ${ }^{21}$.

Inoltre la tab. 2 attesta quanto l'area dei cattolici praticanti sia socialmente periferica (frequenza alla messa più elevata fra le donne, fra i pensionati, ecc.). L'eloquenza dei dati in merito risulta in certa misura stemperata dalla decisione dei commentatori di presentare i dati sulla frequenza alla messa congiuntamente ad altre informazioni, che prescindono dalla dimensione rituale $^{22}$.

${ }^{21}$ Garelli si riferisce a questa quota di popolazione introducendo la nozione di «religione di minoranza» $(1991,16)$. La più alta quota di praticanti rilevata nell'indagine EVSSG è probabilmente da ricondurre alle particolarità del campione, studiato in modo da coprire anche la popolazione dei comuni di montagna, notoriamente più osservanti (Gubert 1992, 19).

22 Per evitare equivoci occorre distinguere fra «rituale» e «ritualistico». Il primo termine deve essere inteso con una connotazione neutra, in quanto designa le pratiche cultuali prescritte da una specifica tradizione religiosa. Il secondo termine contiene una 
TAB. 2. Percentuale di cattolici praticanti regolari in varie categorie di intervistati nel corso di tre sondaggi recenti

\begin{tabular}{lccc}
\hline Variabili e categorie & $\begin{array}{c}\text { Fond. Agnelli } \\
\text { anno 1987 } \\
2423 \text { casi }\end{array}$ & $\begin{array}{c}\text { ISPES } \\
\text { anno 1991 } \\
2000 \text { casi }\end{array}$ & $\begin{array}{c}\text { EVSSG } \\
\text { anno 1991 } \\
2017 \text { casi }\end{array}$ \\
\hline TOTALE PRATICANTI REGOLARI & 29,8 & 29,3 & 39,8
\end{tabular}

SESSO

$\begin{array}{llll}\text { maschi } & 29,6^{*} & 25,8 & 28,8\end{array}$

$\begin{array}{llll}\text { femmine } & 50,3^{*} & 34,6 & 45,4\end{array}$

\section{CONDIZIONE PROFESSIONALE/OCCUPAZIONE}

casalinghe

operai/blue collars

impiegati/white collars

pensionati

in condizione non lavorativa

non occupati

\section{TITOLO DI STUDIO}

\section{elementare}

media

media superiore

laurea

AREA DI RESIDENZA

Nord

Centro

Sud

39,5

21,3

26,7

50,3

$-$

- $\overline{18,3}$

19,1

50,2

$-$

$-$
27,8

$-$

$-$

50,4

51,1

$\begin{array}{lll}- & 43,4 & 39,8 \\ - & 30,3 & 35,0 \\ - & 27,3 & 35,8 \\ - & 27,7 & 38,2\end{array}$

* solo soggetti di età compresa fra 51 e 64 anni, che hanno una pratica religiosa più elevata delle altre classi di età.

Per le fonti vedi tab. 1.

Come si è già notato, Garelli tematizza la differenza tra religione "di minoranza" e "di maggioranza", ma successivamente presenta i dati sulla frequenza ai riti religiosi affiancandoli a quelli relativi alla credenza in Dio, alla fiducia accordata alla

connotazione negativa in quanto si riferisce ai casi in cui il rispetto delle prescrizioni rituali non si fonda su una fede profondamente partecipata ma è dovuto ad un'adesione solo formale e superficiale alle regole, dettata dal conformismo: «un rito senza emozione partecipativa lo è puramente di nome» (Tullio-Altan 1992, 147). 
Chiesa e all'appartenenza a gruppi religiosi (1991, cap. III). Nell'indagine ISPES i dati relativi alla frequenza alla messa vengono analizzati in parallelo (e non giustapposti) a quelli relativi alla preghiera (Brunetta e Longo 1991b, 43-45) e successivamente all' «atteggiamento verso la religione, il concetto di Dio, il posto riservato alla religione nella vita e la credenza nell'aldilà» (L. Sartori 1991, 171). Nell'indagine dell'European Value Systems Study Group (EVSSG) la dimensione rituale viene analizzata congiuntamente ad altre quattro variabili: «importanza della religione nella propria vita, importanza di Dio, frequenza della preghiera, fiducia nella Chiesa come istituzione» (Scartezzini 1992, 485). In questo caso è stata effettuata un'operazione più raffinata - esplicitamente sintattica - che approda ad un indice in base al quale gli intervistati vengono distinti in cinque classi (atei convinti, non religiosi, cattolici con religiosità debole, cattolici osservanti e militanti) (Scartezzini 1992, 486). Il prezzo pagato in termini semantici appare tuttavia eccessivo: $i$ dati relativi a un comportamento (la frequenza in chiesa) vengono irrimediabilmente fusi con altri relativi ad opinioni e atteggiamenti.

Per sintetizzare, a tutte queste ricerche è comune una carenza di riflessione semantica sul nesso concetti-indicatori, che fa perdere di vista lo scarto esistente fra il piano della pratica religiosa e quello delle opinioni, delle credenze e degli atteggiamenti.

\section{Pratica religiosa e fiducia nella Chiesa}

Questa carenza di riflessione semantica è indotta dalla specificità dello strumento utilizzato, e in particolare dal ruolo esclusivo della mediazione linguistica nella rilevazione dei dati mediante interviste strutturate. Non distinguere adeguatamente fra dati relativi alla pratica religiosa (che sono quelli più precisi nel circoscrivere i contorni del mondo cattolico) e dati relativi a opinioni e atteggiamenti sulla Chiesa e sul suo ruolo preclude la possibilità di mettere in piena luce il punto che emerge nitidamente dalle stesse ricerche: a fronte della contrazione del numero dei cattolici praticanti esiste un orientamento generalmente positivo nei confronti della Chiesa cattolica.

In particolare la Chiesa ispira più fiducia delle istituzioni repubblicane, e soprattutto dei partiti politici e dei loro esponen- 
ti. Questo quadro non è il frutto delle recenti vicende giudiziarie degli uomini politici, ma è documentato almeno per tutto il decennio precedente. La tab. 3 mostra come la Chiesa goda di una fiducia assai più elevata rispetto al governo, al parlamento e ai partiti. Nello stesso senso vanno molti altri dati presentati nelle indagini citate, come l'ampio consenso che circonda le prese di posizione dei rappresentanti della Chiesa contro la mafia (Brunetta e Longo 1991b, 80), contro la discriminazione razziale e a favore del disarmo (Scartezzini 1992, 477).

Il punto culturalmente e politicamente decisivo è, ovviamente, il divario di legittimità e di fiducia che separa la Chiesa cattolica dalle istituzioni statuali e che gli avvenimenti di questi mesi non contribuiscono certo a ridurre. Questo divario non è confinato alle risposte ai vari questionari ma si risolve in scelte concrete da parte dei cittadini, come ad esempio avviene nella destinazione dell'otto per mille da indicare nella dichiarazione dei redditi. Secondo i dati del Ministero delle Finanze, citati da Margiotta-Broglio, il $75 \%$ dei contribuenti che hanno esplicitato la loro scelta ha indicato nella Chiesa cattolica il destinatario dei contributi, contro il $25 \%$ che le ha destinate alle attività sociali e umanitarie gestite dallo Stato (Margiotta-Broglio 1991, 314). Un più recente sondaggio Doxa, commissionato dalla Conferenza episcopale, indica che solo il $45 \%$ di coloro che hanno firmato a favore della Chiesa è costituito da cattolici praticanti ${ }^{23}$.

Questa tensione fra ridotto numero di praticanti e larga fiducia nella Chiesa come istituzione, a parere di chi scrive, costituisce uno degli aspetti più rilevanti dell'attuale congiuntura politica e culturale; ed è sullo sfondo di questi due fenomeni che potranno essere giudicate le varie iniziative politiche che vedono come protagonisti esponenti cattolici, e richiamate di passata all'inizio di questo saggio.

Naturalmente il successo di queste iniziative sarà condizionato, anche se non soprattutto, dall'immediato retroterra di cattolici militanti su cui queste iniziative potranno contare, soprattutto nelle fasi iniziali. Ma è proprio su questo punto che le indagini per sondaggio presentano un altro punto di debolezza, anch'esso imputabile a una fondamentale carenza di riflessione semantica sulla proprietà «pratica religiosa» e la conseguente concettualiz-

${ }^{23}$ Cfr. «Avvenire» del 24 giugno 1992, p. 13. 
TAB. 3. Grado di fiducia espresso nei confronti di varie istituzioni rilevato in vari sondaggi. Percentuale di soggetti che nutrono «molta» o «abbastanza» fiducia nelle istituzioni indicate

\begin{tabular}{|c|c|c|c|c|c|}
\hline $\begin{array}{l}\text { campione } \\
\text { numero casi }\end{array}$ & $\begin{array}{l}\text { VSSG } 198 \\
\text { elettori } \\
1348\end{array}$ & $\begin{array}{l}\text { IARD } 1983 \\
\text { giovani } \\
4000 \text { circa }\end{array}$ & $\begin{array}{l}\text { IARD } 1987 \\
\text { giovani } \\
2000 \text { circa }\end{array}$ & $\begin{array}{c}\text { F. Agnelli } 87 \\
\text { elettori } \\
2423\end{array}$ & $\begin{array}{c}\text { EVSSG } 1991 \\
\text { elettori } \\
2017\end{array}$ \\
\hline Polizia & 68 & 70 & 71 & - & 66 \\
\hline Carabinieri & - & 64 & 63 & - & - \\
\hline Industrie private/grandi & di 33 & - & - & 74 & 62 \\
\hline Scuola/insegnanti & 56 & 70 & 68 & 69 & 48 \\
\hline Chiesa/sacerdoti & 60 & 40 & 40 & 60 & 63 \\
\hline Giornali e TV & 31 & - & - & 63 & 39 \\
\hline Magistratura & 43 & 53 & 51 & 51 & 31 \\
\hline Forze armate/militari & 58 & 41 & 39 & - & 48 \\
\hline Industrie pubbliche & - & - & - & 50 & - \\
\hline Sindacato/sindacalisti & 28 & 31 & 24 & 24 & 34 \\
\hline Parlamento & 31 & - & - & - & 32 \\
\hline Funzionari pubblici & 28 & 26 & 28 & - & 27 \\
\hline Governo & - & 26 & 38 & 20 & - \\
\hline Uomini politici & - & 17 & 21 & - & _ \\
\hline Partiti & - & - & - & 12 & - \\
\hline
\end{tabular}

Fonti: per le due indagini IARD cfr. Cavalli e De Lillo (1988, 189); per la ricerca della Fondazione Agnelli cfr. Garelli $(1991,75)$; per l'indagine EVSSG del $1981 \mathrm{cfr}$. Calvaruso e Abbruzzese (1985); per quella più recente cfr. Gubert $(1992,605)$.

zazione di questa come proprietà continua. Essa viene così definita operativamente mediante il ricorso ad una classificazione con categorie ordinate, che vanno da «tutte le domeniche o più» a «mai», passando per varie gradazioni intermedie.

Questa concettualizzazione, se consente di rilevare la componente di popolazione che non si reca mai in chiesa, impedisce però di cogliere pienamente quella differenza fra «cattolici conformisti», «praticanti» e «devoti» che è stata messa in rilievo da LeBras (1969) e che per lo scienziato sociale è cruciale. Solo questa differenza consente infatti di distinguere fra una reale appartenenza cattolica e un'osservanza puramente ritualistica, priva di partecipata convinzione, spesso dettata unicamente dalla persistente influenza simbolica della Chiesa nel «marcare» gli snodi cruciali della vita degli individui (battesimo, matrimonio ecc.) ${ }^{24}$.

${ }^{24}$ L'elevato ricorso ai riti religiosi in corrispondenza di questi snodi cruciali è da ricondurre alla natura rassicurante del rito di fronte ad un «passaggio» che come tale viene vissuto come minaccia e pericolo. Sul significato antropologico dei riti di passaggio è sempre valido il classico Van Gennep (1909); per una trattazione più recente cfr. 
Ma una classificazione con categorie ordinate è adatta a rilevare differenze di grado, non consente di rilevare differenze di specie, quali quelle che si pongono fra i diversi tipi di «cattolici», ad esempio fra «conformisti stagionali» e «praticanti». In particolare, una definizione operativa della pratica religiosa di natura tipologica consentirebbe di mettere in rilievo la differente caratterizzazione in senso ritualistico del cattolicesimo meridionale rispetto a quello settentrionale.

L'accentuazione ritualistica e conformista del cattolicesimo meridionale ( $\mathrm{ma}$ non solo meridionale, come vedremo) è un tratto culturale noto da tempo, ripetutamente rilevato dagli osservatori, da Gramsci e Levi fino a De Martino, Di Nola e Galasso (Cartocci 1990, 176-177). Del resto anche recentemente questa accentuazione conformista e ritualistica è stata denunciata dai vescovi italiani. Nel loro già richiamato documento sulla situazione sociale, politica ed economica del Mezzogiorno si legge che la «nuova evangelizzazione [non deve tendere] al soffocamento delle manifestazioni della "pietà popolare", ma soltanto alla sua purificazione. Senza questa purificazione... la pietà popolare può ridursi a essere domanda senza risposta, croce senza risurrezione, gestualità senza contenuti, memoria di pure emozioni» ${ }^{25}$.

In un periodo in cui la Chiesa sta assumendo un nuovo ruolo politico-culturale, facendosi interprete di esigenze di integrazione insoddisfatte dalle istituzioni pubbliche, la diversa religiosità che contraddistingue le varie zone dell'Italia potrebbe portare a ulteriori differenze e a diversi esiti, anche politici. A questo punto il condizionale è d'obbligo, dal momento che il processo è in pieno svolgimento e aperto a vari sbocchi; resta il fatto che le indagini condotte mediante sondaggi non ci sono di alcun aiuto per approfondire questa tematica. Esse sono infatti quasi del tutto mute in merito a questa differenza fra gli

Turner (1986) e Tullio-Altan (1992, 142-145). Naturalmente su questo più profondo significato antropologico si sono innestate motivazioni sociali, più o meno autentiche, fino alle più recenti degenerazioni consumistiche (Longo 1991, 167).

${ }^{25}$ Cfr. Chiesa italiana e Mezzogionno: sviluppo nella solidarietà, Torino, Edizioni Paoline, 1989, p. 26 (corsivi miei). Anche recentemente il vescovo di Nola ha denunciato, nella sua lettera pastorale, «certe abitudini e tradizioni [secondo cui] in certe zone della diocesi continuino chilometriche processioni in cui di sacro non c'è altro che l'immagine di un santo e la presenza puramente decorativa del prete» (cfr. «Avvenire» dell'8 novembre 1992, p. 13). 
orientamenti cattolici di massa prevalenti nelle diverse aree del paese $^{26}$.

D'altra parte, come risulta evidente dalla tab. 2, le varie indagini (al di là di alcune importanti differenze) attestano invece come le differenze in termini di pratica religiosa siano molto più sensibili ai fattori demografici e sociali che a quelli geografici. Le differenze fra maschi e femmine ${ }^{27}$, o fra operai e pensionati, sono sistematicamente assai più elevate di quelle fra le diverse zone del paese. In altri termini, la categoria dei praticanti regolari sarebbe fortemente differenziata dal resto della popolazione per sesso, età e professione ma non per area di residenza. Un risultato decisamente sorprendente in un paese in cui non esiste variabile (demografica, economica, sociale) che non presenti un'accentuata articolazione regionale! ${ }^{28}$ Ancor più sorprendente alla luce delle già ricordate differenze fra gli orientamenti religiosi del Nord e del Sud.

\section{Gli indicatori di pratica religiosa}

Alla luce di quanto esposto nella sezione precedente, il ricorso a dati aggregati è il solo modo per supplire alla carenza di dati di sondaggio disponibili per le diverse zone del paese. Questi dati consentono inoltre di accertare la presenza differenziale dei «devoti», dei «praticanti» e dei «conformisti» non rilevata dai sondaggi.

Già nel precedente lavoro dedicato alla geografia della seco-

${ }^{26}$ L'indagine condotta dall'ISPES è, da questo punto di vista, meno criticabile di altre, dal momento che l'accentuazione ritualista dei cattolici meridionali viene rilevata, anche se di sfuggita e con indicatori eccentrici rispetto al cuore del problema (Brunetta e Longo 1991b, 24).

${ }_{27}$ Una interessante interpretazione della più elevata pratica religiosa che contraddistingue le donne è stata recentemente avanzata da Fanny Cappello (1992). Da questo lavoro si desume peraltro che la forbice fra maschi e femmine tende a ridursi nel tempo.

${ }^{28}$ Ciò è dovuto, oltre ai citati limiti dello strumento-intervista strutturata nel rilevare la proprietà, anche al modo in cui vengono definite le grandi ripartizioni geografiche. In questi come in molti altri casi, la ripartizione è stata effettuata senza tener conto delle note differenze - proprio in termini di orientamenti cattolici - tra le diverse regioni del Nord. Si spiega così perché l'area contrassegnata da una più ridotta pratica religiosa risulti sistematicamente il Centro, costituito da tre regioni «rosse» e dal Lazio, all'interno del quale è preponderante il peso delle interviste effettuate a Roma (e che dunque riflettono il clima secolarizzato della metropoli). Al contrario il Nord è l'area più eterogenea, ma i suoi valori medi risultano vicini a quelli del Sud. 
larizzazione ero ricorso a due indicatori di pratica religiosa disponibili per le singole province. $\mathrm{Da}$ un lato la percentuale di matrimoni civili consentiva di rilevare l'ammontare, provincia per provincia, della quota dei non cattolici. A questo indicatore tuttavia occorreva affiancarne un altro, al fine di differenziare «devoti» e «praticanti» dai «conformisti».

L'indicatore che ho utilizzato a questo scopo per il 1981 è stato la diffusione di «Famiglia Cristiana», all'epoca il settimanale più venduto in Italia, che veniva distribuito in gran parte mediante la rete delle parrocchie e costituiva quindi - a livello di massa - un termometro dell'intensità dell'adesione ai valori cattolici, al di là della mera frequenza alla messa.

I legami «sintattici» fra i due indicatori erano inizialmente risultati deboli e di segno opposto a quello atteso $(r=+0,10)$, imponendo un supplemento di riflessione «semantica». In effetti la diffusione di «Famiglia Cristiana» dipendeva anche dalla diffusione complessiva dei settimanali. L'indicatore conteneva cioè una «parte estranea» ${ }^{29}$ che lo connetteva semanticamente alla propensione alla lettura, e dunque al reddito medio nella provincia. Esclusa questa parte estranea ${ }^{30}$, il coefficiente di correlazione con il tasso di matrimoni civili ha assunto l'atteso segno negativo, ma con un valore di $r$ decisamente debole $(r=-0,34)$, che attesta un'insufficiente congruenza fra gli indicatori.

L'ulteriore esplorazione, di natura sintattica, della parentela statistica fra i due indicatori ha quindi permesso di accertare le differenze fra Nord e Sud dal punto di vista della pratica religiosa. Infatti nelle aree centro-settentrionali ${ }^{31}$ la relazione tra $\mathrm{i}$ due indicatori era risultata forte e negativa (come nelle attese)

${ }^{29}$ La «parte estranea» è il contenuto di informazione dell'indicatore che non è comune alla proprietà che si intende rilevare (Marradi 1984a, 36). In termini linguistici è assimilabile a un «rumore». Tanto più elevata è questa parte, tanto meno valido è l'indicatore.

${ }^{30}$ Questa parte estranea è stata esclusa calcolando i residui della regressione della diffusione di «Famiglia Cristiana» sulla diffusione complessiva dei settimanali più venduti.

${ }^{31}$ Le aree in cui sono state suddivise le province sono quelle definite da Arculeo e Marradi (1985). Il Nord Laico comprende il Piemonte, la Liguria, il Friuli-Venezia Giulia e le province di Milano, Varese, Pavia, Cremona, Rovigo, Venezia e Belluno. La Zona Bianca comprende le restanti province venete, Trento, Como, Sondrio, Bergamo, Brescia e l'enclave di Lucca. La Zona Rossa è costituita da Emilia-Romagna, Toscana (meno Lucca), Umbria e dalle province di Mantova, Pesaro e Ancona. Il Centro comprende Lazio, Abruzzo, Marche meridionali e Sardegna. Tutte le altre regioni del meridione continentale e la Sicilia costituiscono il Sud. Aosta e Bolzano sono escluse per le loro specificità linguistiche e politiche. 
TAB. 4. Correlazione calcolata fra il tasso di matrimoni civili e la diffusione di «Famiglia Cristiana», al lordo e al netto della diffusione dei settimanali, nel 1981

\begin{tabular}{lccc}
\hline Zona $^{*}$ & $\begin{array}{c}\text { numero } \\
\text { province }\end{array}$ & al lordo & $\begin{array}{c}\text { al netto } \\
\text { (residui) }\end{array}$ \\
\hline Nord Laico & 21 & $-0,75$ & $-0,82$ \\
Zona Bianca & 10 & $-0,68$ & $-0,74$ \\
Zona Rossa & 21 & $-0,41$ & $-0,78$ \\
Centro & 15 & $-0,03$ & $-0,56$ \\
Sud & 26 & $-0,08$ & $-0,23$ \\
ITALIA & 93 & $+0,10$ & $-0,34$ \\
\hline
\end{tabular}

* Le zone sono quelle definite da Arculeo e Marradi (1985). Sono escluse Aosta e Bolzano.

mentre nelle due aree centro-meridionali restava negativa ma con un valore di $r$ sensibilmente inferiore, tale da deprimere la forza della relazione calcolata sull'insieme delle province (tab. 4).

In particolare, la ridotta congruenza nell'estremo Sud attestava l'esistenza in quest'area di un cattolicesimo diversamente caratterizzato rispetto a quello centro-settentrionale, e segnato da una forte prevalenza di elementi conformisti e ritualisti (attestata dal ridotto numero di matrimoni civili) rispetto a quelli più intensamente partecipati (ridotta diffusione di «Famiglia Cristiana», anche scontando il divario in termini di diffusione dei settimanali in generale).

A parte il caso delle regioni dell'estremo Sud, la congruenza fra i due indicatori nelle altre quattro aree mi aveva persuaso a costruire un indice di «secolarizzazione» (intesa come recessione dell'influenza della Chiesa a livello di massa), la cui distribuzione è riportata nella fig. $4^{32}$.

Come si osserva dalla figura, il grado di secolarizzazione delle varie province dipendeva in buona misura dall'intersecarsi di tre dimensioni:

1) la presenza di una tradizione cattolica oppure socialista;

2) la presenza di metropoli;

3) l'accesso al mare e la collocazione lungo le grandi vie di comunicazione. Significativo a questo proposito che la regione con il grado di secolarizzazione più elevato sia risultata la Ligu-

32 L'indice è stato costruito sommando ai punteggi standardizzati del tasso di matrimoni civili l'opposto dei punteggi standardizzati dei residui della regressione di $\ll \mathrm{Fa}$ miglia Cristiana» sulla diffusione media dei settimanali (Cartocci 1990, 177). 


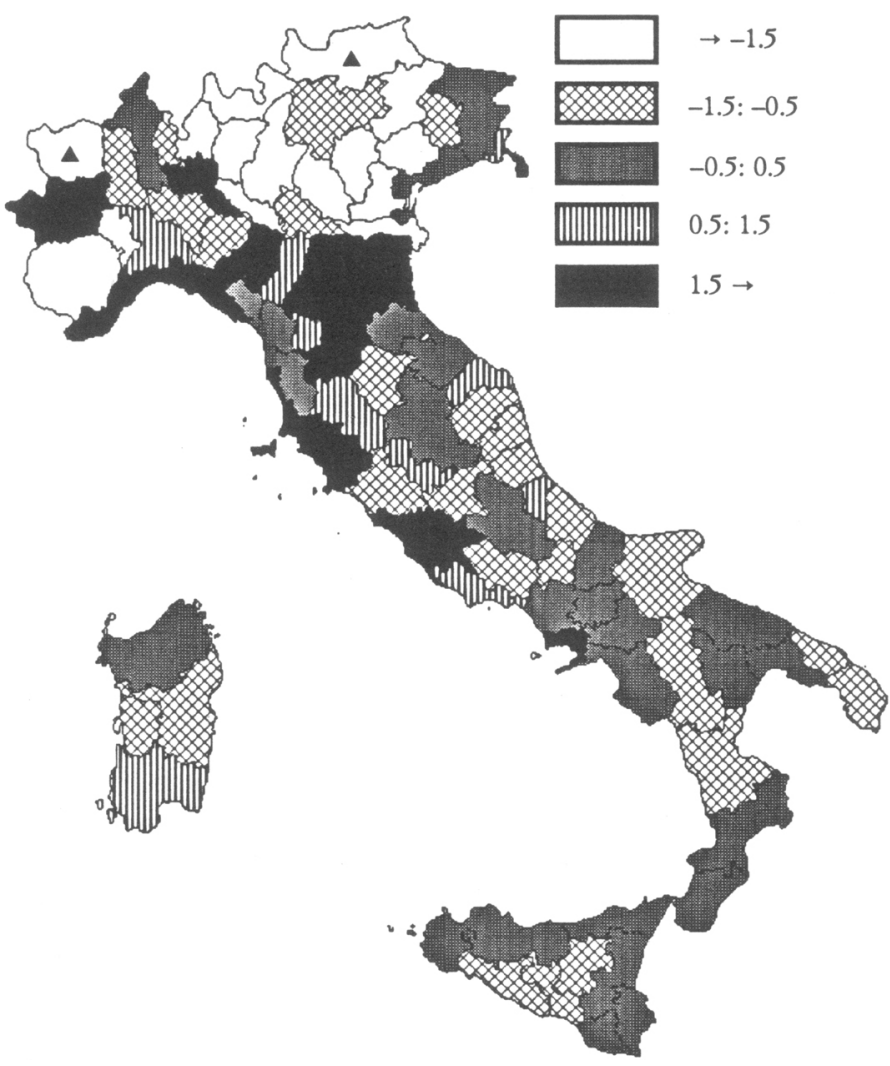

FIG. 4. Il livello di secolarizzazione in 93 province nel 1981. Sono escluse Aosta e Bolzano

ria. Non meno interessante, a mio parere, il trasparire di antiche tradizioni mercantili e cosmopolite: $i$ vecchi porti franchi di Trieste e Livorno risaltavano come le aree più secolarizzate, a conferma di quanto era emerso anche in occasione del referendum sul divorzio; cfr. Marradi (1974) e Tullio-Altan (1974).

Alla luce della ridotta congruenza fra i due indicatori nelle regioni meridionali, l'applicazione in quest'area dello stesso indice additivo utilizzato per il Centro-Nord era «sostanzialmente indebita», dovuta alla necessità di disporre di un unico strumento da confrontare con variabili di natura elettorale (Cartocci 1990, 186). Un indice additivo messo a punto per rilevare differenze di grado non poteva che obnubilare le differenze $d i$ 
specie fra le caratteristiche della pratica religiosa del CentroNord e quelle del Centro-Sud.

\section{Geografia della pratica religiosa nel 1991}

Questa differenza fra le varie aree del paese si è confermata per tutti gli anni ottanta. Ho infatti ripetuto la rilevazione con gli ultimi dati disponibili per i medesimi indicatori, cioè per la diffusione di «Famiglia Cristiana» e degli altri settimanali e per il tasso di matrimoni civili, tutti relativi al $1991^{33}$.

Il processo di secolarizzazione (inteso sempre come recessione dell'influenza cattolica a livello di massa) viene attestato da entrambi gli indicatori, a livello nazionale e in quattro zone su cinque (tab. 5).

Come si vede, in un decennio il numero di matrimoni civili è notevolmente aumentato. Più ridotta la contrazione della diffusione di «Famiglia Cristiana». A questo proposito occorre però avvertire il lettore che il processo di secolarizzazione è evidente anche per quanto riguarda la diffusione del settimanale cattolico, al di là del numero di copie vendute. Infatti esso ha perduto il primato della diffusione, a favore di una testata di argomento televisivo $^{34}$. Inoltre il processo di secolarizzazione si avverte nelle

TAB. 5. Valori medi provinciali del tasso di matrimoni civili nel 1981 e nel 1991 e della diffusione di «Famiglia Cristiana», nel 1981 e nel 1991

\begin{tabular}{lrrrrrrr}
\hline Zona $^{\star}$ & \multicolumn{3}{c}{ tasso di matrim. civili } & \multicolumn{3}{c}{ diffus. «Famiglia Cristiana»** } \\
& 1981 & 1991 & Differ. & 1981 & 1991 & Differ. \\
\hline Nord Laico & 16,0 & 23,5 & 7,5 & & & & \\
Zona Bianca & 8,8 & 15,3 & 6,5 & 55,6 & 28,5 & $-7,1$ \\
Zona Rossa & 15,1 & 21,0 & 5,9 & 20,1 & 39,2 & $-11,9$ \\
Centro & 8,3 & 15,2 & 6,9 & 18,0 & 14,6 & $-1,7$ \\
Sud & 6,4 & 9,2 & 2,8 & 6,1 & 8,2 & $+2,1$ \\
ITALIA & 11,1 & 16,7 & 5,7 & 22,1 & 19,4 & $-2,7$ \\
\hline
\end{tabular}

* Le zone sono quelle definite da Arculeo e Marradi (1985). Sono escluse Aosta e Bolzano.

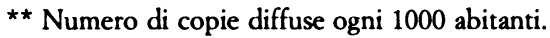

${ }^{33}$ I dati relativi al tasso di matrimoni civili sono tratti dal «Bollettino mensile di statistica», LXI, n. 6, del giugno 1992. I dati relativi a «Famiglia Cristiana» e agli altri settimanali utilizzati per calcolare i residui («Sorrisi e Canzoni TV», «Oggi», «Gente» e «Panorama») sono tratti da «ADS Notizie», n. 62, ottobre-dicembre 1992.

34 Si tratta di «Sorrisi e canzoni TV» che ha diffuso 2.296 .425 copie contro 
modalità stesse di diffusione di «Famiglia Cristiana», che oggi viene venduto anche nelle edicole, e dunque ha subito un processo di omologazione che non può essere interpretato che come un effetto della riduzione della pratica religiosa.

Il processo di secolarizzazion $e^{35}$ ha però avuto in questo decennio una traiettoria diversa nelle varie aree del paese. Particolarmente intensa è stata l'erosione dei valori cattolici nella Zona Bianca. La Zona Rossa presenta invece una dinamica meno intensa rispetto alle altre aree del Centro-Nord, presumibilmente a causa di un effetto-tetto. Le regioni meridionali sono state investite da questa corrente in misura molto minore di tutte le rimanenti: il tasso di matrimoni civili è cresciuto meno della metà della media nazionale; «Famiglia Cristiana», a fronte di un generale arretramento nelle regioni centro-settentrionali, ha leggermente aumentato la sua presenza nel Sud, che resta peraltro assai contenuta.

Forse proprio la «secolarizzazione» dei canali di distribuzione di «Famiglia Cristiana» ha consentito alla testata di aumentare (o non ridurre, come al Centro) la sua diffusione nelle zone di minore penetrazione. In tema di riflessione «semantica» sul nesso fra la proprietà generale e questo indicatore, ciò significa che questa decisione di marketing ha prodotto un allentamento del giunto semantico, confermato dalla più ridotta congruenza di questo indicatore con il tasso di matrimoni civili (tab. 6).

Nell'insieme delle province, in dieci anni è aumentata la relazione positiva, e dunque contraria alle attese, fra tasso di matrimoni civili e diffusione «lorda» di «Famiglia Cristiana» ( $r$ è salito da $+0,10 a+0,28)$. È invece diminuita la relazione con la diffusione «netta» del settimanale, ottenuta cioè escludendo la propensione alla lettura $(r$ è sceso da $-0,34$ a $-0,19)$.

1.058.078 di «Famiglia Cristiana». La diffusione delle altre testate utilizzate è molto più ridotta. Varia dalle 890.512 di «Gente» alle 466.872 di «Panorama». Sulle trasformazioni del mercato dei settimanali nel corso degli anni ottanta, e in particolare sull'espansione delle testate che trattano principalmente di argomenti e programmi televisivi, cfr. Peri (1988).

${ }^{35}$ Vari altri indicatori potrebbero utilmente essere analizzati, da quelli più tradizionali - come il tasso di natalità, il numero di figli nati fuori dal matrimonio, il numero di divorzi, separazioni e aborti (cfr. Nesti 1992) - a quelli previsti dal nuovo Concordato, come la destinazione dell'otto per mille e le erogazioni fiscalmente deducibili (cfr. Margiotta-Broglio 1991). Ho utilizzato solo i due precedenti perché mi sembrano premature le operazioni sintattiche necessarie per «gestire» indicatori più numerosi. I due utilizzati mi sembrano relativamente chiari e consentono un puntuale confronto intertemporale. 
TAB. 6. Correlazione fra il tasso di matrimoni civili e la diffusione di «Famiglia Cristiana», al lordo e al netto della diffusione dei settimanali, nel 1991

\begin{tabular}{lccc}
\hline Zona $^{\star}$ & $\begin{array}{c}\text { numero } \\
\text { province }\end{array}$ & al lordo & $\begin{array}{c}\text { al netto } \\
\text { (residui) }\end{array}$ \\
\hline Nord Laico & 21 & $-0,64$ & $-0,83$ \\
Zona Bianca & 10 & $-0,66$ & $-0,65$ \\
Zona Rossa & 21 & $-0,36$ & $-0,63$ \\
Centro & 15 & $+0,15$ & $-0,29$ \\
Sud & 26 & $-0,22$ & $-0,21$ \\
ITALIA 1991 & 93 & $+0,28$ & $-0,19$ \\
ITALIA 1981 & 93 & $+0,10$ & $-0,34$ \\
\hline
\end{tabular}

* Le zone sono quelle definite da Arculeo e Marradi (1985). Sono escluse Aosta e Bolzano.

Apparentemente il nostro paese è diventato in dieci anni ancora meno comprensibile: si vende più «Famiglia Cristiana» dove ci si sposa meno in chiesa. È peraltro da escludere che si tratti di un'immagine distorta da un indicatore usurato dal tempo. L'indagine ISPES indica che tra i cattolici più attivi è la testata di gran lunga più diffusa tra quelle di orientamento religioso (Brunetta e Longo 1991b, 36).

In termini di relazioni «sintattiche» fra i due indicatori, si tratta di un nitido «effetto compositivo»: a livello nazionale risulta una relazione che è dovuta alla sovrapposizione di relazioni di segno opposto all'interno delle varie aree (Arculeo e Marradi 1985 ). In dieci anni la distanza fra le aree centro-settentrionali e quelle centro-meridionali si è allargata, come sintetizza la tab. 7. A nord della linea che unisce la foce della Fiora alla foce del Tronto la correlazione fra $\mathrm{i}$ due indicatori assume un valore quattro volte più elevato che nelle altre regioni. Questa drastica caduta della congruenza fra $\mathrm{i}$ due indicatori in una metà del paese rende impossibile la costruzione di un indice additivo, analogo a quello messo a punto con gli stessi indicatori riferiti al 1981, nel presupposto che la pratica religiosa fosse concettualizzabile come una proprietà continua.

La dinamica dei due indicatori ha chiarito in questo decennio che la pratica religiosa è meglio rilevabile ricorrendo a uno strumento che colga non tanto differenze di grado, quanto differenze di specie. La via da percorrere è dunque quella di un indice tipologico, da costruire mediante il prodotto logico, e non l'addizione, fra i due indicatori.

Alla luce della disposizione delle 93 province sul piano car- 
TAB. 7. Correlazione fra il tasso di matrimoni civili e la diffusione netta di «Famiglia Cristiana», nel 1981 e nel 1991

\begin{tabular}{lccc}
\hline Zona* & $\begin{array}{c}\text { numero } \\
\text { province }\end{array}$ & 1981 & 1991 \\
\hline Nord-Centro & 54 & $-0,69$ & $-0,63$ \\
Centro-Sud & 39 & $-0,33$ & $-0,15$ \\
ITALIA & 93 & $-0,34$ & $-0,19$ \\
\hline
\end{tabular}

* Il Nord-Centro comprende le regioni settentrionali più la Toscana, l'Umbria e le Marche; il Centro-Sud tutte le altre regioni. Sono escluse Aosta e Bolzano.

tesiano definito dai due indicatori (fig. 5), è possibile osservare le ragioni della loro scarsa correlazione. Infatti solo una parte delle province si colloca lungo la linea che collega le province dell'area bianca (Como, Bergamo, Vicenza, ecc.), dove a valori bassi del tasso di matrimoni civili corrisponde un'elevata diffusione di «Famiglia Cristiana», a quelle più secolarizzate (Trieste, Bologna, Livorno, ecc.). Un terzo delle province, tra cui quasi tutte quelle del Sud, si colloca invece al di sotto di questa

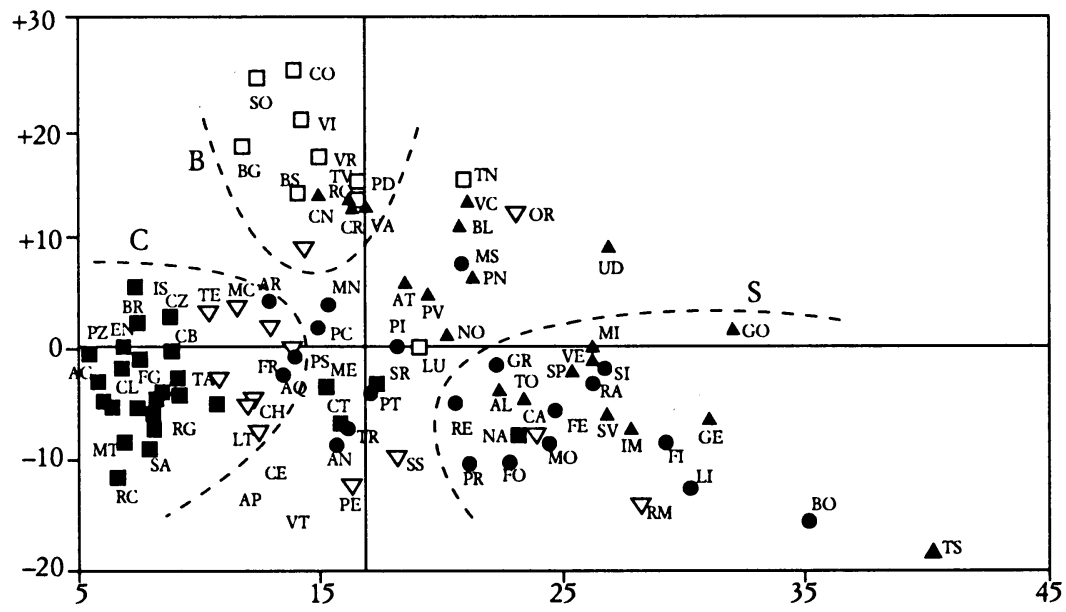

FIG. 5. Collocazione di 93 province in base al tasso di matrimoni civili (asse orizzontale) $e$ alla diffusione netta di «Famiglia Cristiana» (asse verticale) nel 1991. I valori della diffusione netta di «Famiglia Cristiana» sono intorno a 0 in quanto sono residui della regressione sulla diffusione di media dei settimanali. Sono escluse Aosta e Bolzano 
direttrice, con tassi di matrimoni civili anche più bassi delle province «bianche» e con una diffusione di «Famiglia Cristiana» analoga a quella delle province più secolarizzate.

Le curve tratteggiate isolano e raggruppano questi tre tipi di province che rivelano forme di pratica religiosa radicalmente diverse. Le province racchiuse dalla linea contrassegnata da una $\mathrm{S}$ sono le 24 province più secolarizzate, con tassi di matrimoni civili molto elevati $e$ ridotta diffusione di «Famiglia Cristiana». Si tratta di tutte le province metropolitane e di buona parte di quelle della Zona Rossa, contrassegnate nella figura da un disco nero. Inoltre la capacità secolarizzante dell'accesso al mare o lungo le vie di comunicazione spiega la presenza in questo gruppo delle province litoranee della Liguria e dell'alto Adriatico, di Alessandria e Cagliari.

All'estremo opposto, circoscritte dalla linea $B$, vi sono 11 province del Lombardo-Veneto bianco - da Varese a Treviso con l'aggiunta di Cuneo e Nuoro. È questa l'area in cui la pratica religiosa cattolica è più intensa e partecipata, con la massima diffusione relativa di «Famiglia Cristiana» e un ridotto tasso di matrimoni civili. La linea $\mathrm{C}$ circoscrive il gruppo più compatto e numeroso di province (in tutto 34), in cui prevale un tipo di religiosità più conformista. In questo gruppo troviamo, oltre a quasi tutte le province del Centro (triangoli bianchi) e del Sud (quadrati neri), anche tre della Zona Rossa (Arezzo, Pesaro e Perugia). All'interno di questa vasta categoria resta peraltro sensibile la differenza che separa le province dell'estremo Sud, raggruppate a ridosso del margine sinistro della figura, da quelle del Centro (e dalle tre della Zona Rossa sopra menzionate), collocate a destra delle precedenti.

Naturalmente questa suddivisione, effettuata manualmente, senza ricorrere a procedure di clustering, contiene indubbi elementi di arbitrarietà. I tre gruppi di province avrebbero potuto essere identificati - altrettanto legittimamente - tracciando linee diverse. Inoltre per attenuare l'arbitrarietà ho previsto una sorta di area cuscinetto, una categoria residuale in cui ricadono tutte quelle province che non è stato possibile attribuire con certezza ai tipi delle province secolarizzate, cattoliche o conformiste, e in cui potrebbero eventualmente ricadere quei casi che sono ai margini delle tre aree circoscritte. Tutte le categorie residuali sono fortemente eterogenee: questa lo è dal punto di vista della distribuzione geografica. Vi troviamo province padane interposte fra aree rosse e bianche (Mantova, Pavia, Piacenza), province 


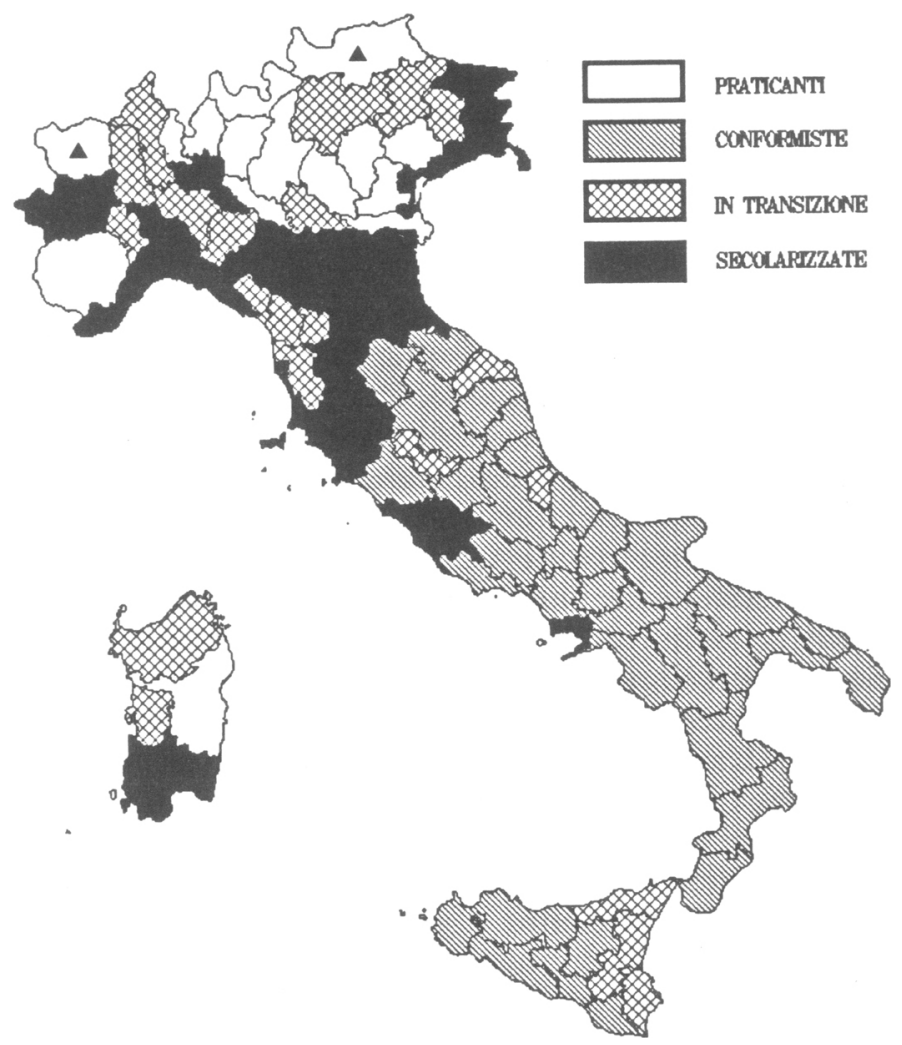

FIG. 6. Tipologia delle 93 province secondo la pratica religiosa nel 1991. Sono escluse Aosta e Bolzano

minori della Zona Rossa, aree metropolitane e dinamiche del Centro e del Sud (Catania, Messina, Siracusa, Sassari e Pescara).

La rappresentazione cartografica di questa tipologia è riportata nella fig. 6, che ne evidenzia meglio le articolazioni territoriali. Il Centro-Nord è la ripartizione più frastagliata, con l'ampia area cattolica del Lombardo-Veneto circondata da vaste zone secolarizzate. A sud della Romagna (sul versante adriatico) e di Grosseto (sul versante tirrenico) inizia la vasta zona del cattolicesimo conformista, da cui si staccano - come ricordato sopra - alcune province costiere del Centro e del Sud. Netta risalta poi la condizione di «insularità» delle due province metropolitane di Roma e Napoli. Una considerazione a parte merita 
la Sardegna, la cui polarizzazione interna richiama quella che caratterizza le regioni settentrionali, a conferma della sua irriducibilità alle altre regioni centro-meridionali.

\section{Per concludere}

Un'analisi delle caratteristiche culturali del nostro paese appare quanto mai urgente in una congiuntura delicata come quella che stiamo attraversando. Per far questo occorrono strumenti adeguati, precisi e sensibili. A fronte della disponibilità di risorse di calcolo potenti e di facile accesso, l'attuale produzione di dati, aggregati e individuali, da parte di agenzie pubbliche $\mathrm{e}$ private, può indirizzare $\mathrm{i}$ ricercatori a esplorare la via delle parentele sintattiche piuttosto che quella delle connessioni semantiche. Nelle pagine precedenti ho cercato di illustrare come, in riferimento alla rilevazione della pratica religiosa, alcune ricerche recenti, condotte mediante sondaggi, soffrano di vari limiti. Una carenza di riflessione semantica ha impedito di conferire il dovuto rilievo agli indicatori di pratica religiosa rispetto ad opinioni e atteggiamenti. D'altra parte proprio questa carenza di riflessione semantica porta a rilevare la pratica religiosa ricercando differenze di grado (frequenza alla messa più o meno assidua) quando invece sarebbe più opportuno rilevare differenze di specie, tali da mettere in evidenza le diverse forme di religiosità presenti nel paese.

Il ricorso a indicatori aggregati a livello provinciale (tasso di matrimoni civili e diffusione di «Famiglia Cristiana») consente invece di accertare la prevalenza di una forma di cattolicesimo ritualista e conformista nelle regioni centro-meridionali, che si contrappone da un lato al cattolicesimo più partecipato del Lombardo-Veneto e dall'altro alla forte secolarizzazione che caratterizza le metropoli, le province «rosse» e le aree costiere del Nord.

D'altra parte, nell'ultimo decennio il processo di secolarizzazione ha proceduto molto più rapidamente nelle regioni centro-settentrionali che non in quelle centro-meridionali, allargando ulteriormente le differenze e imponendo una revisione dell'indice utilizzato in precedenza. Se nel 1981 era ancora legittimo costruire un indice additivo, che rilevava il grado di secolarizzazione delle 93 province, i dati relativi al 1991 attestano una forte riduzione della congruenza tra gli indicatori nella metà 
centro-meridionale del paese, tale da imporre una diversa costruzione dell'indice. Questa volta i due indicatori sono stati combinati in un indice tipologico, che ha permesso di evidenziare le differenze di specie fra tre tipi di province e di individuare alcune province di transizione, in cui il processo secolarizzante è in atto ma non esplica ancora effetti elevati.

Sul piano della congiuntura politica e culturale che il nostro paese si trova ad affrontare - e alla quale sono stati fatti solo accenni fugaci - la geografia della secolarizzazione descritta nella sezione precedente consente di stabilire con maggior precisione tre punti. Innanzitutto risulta ancora più chiara la coincidenza nelle stessa area fra il voto alla Lega Nord e la diffusione dei valori cattolici (Diamanti e Riccamboni 1992; Sani 1993). D'altra parte - ed è questo il secondo punto - una base di massa capace di far decollare i vari tentativi di rinnovamento politico del mondo cattolico pare individuabile esclusivamente in queste stesse aree. Evidentemente anche questi due motivi, di natura politica, hanno contribuito a rendere così aspra la contrapposizione che in questi mesi ha diviso i vertici della Chiesa e quelli della Lega Nord. In terzo luogo, gli appelli alla «speranza e alla solidarietà» lanciati dai vertici della Chiesa possono contare, per realizzare il loro disegno di rinnovamento civile e morale, più sul vasto prestigio della Chiesa come istituzione (e sulla latitanza delle istituzioni statuali nel fornire strumenti efficaci di integrazione sociale) che non sulla quota minoritaria di cattolici che vivono in termini autenticamente partecipati la loro fede.

Sul piano metodologico possiamo trarre la conclusione che l'esplorazione dei legami semantici fra proprietà non direttamente rilevabili e indicatori è un processo che non può mai considerarsi concluso. La dinamica storica modifica continuamente il significato degli indicatori. In Italia, poi, questa stessa dinamica altera in modo sensibile anche i termini delle differenze fra $i$ vari contesti territoriali. Il caso dell'indice di secolarizzazione, esposto nella sezione precedente, chiarisce come parlare di indicatori a geometria variabile non sia solo una formula, di vago sapore ingegneristico, evocata per riverberare sulle nostre incerte procedure d'indagine il prestigio di professionalità più accreditate. In realtà la geometria variabile, ovvero la possibilità di modificare la relazione fra gli indicatori alla luce di nuove considerazioni semantiche, appare un artificio necessario a contrastare la rapida obsolescenza dei nostri strumenti d'indagine. 


\section{Riferimenti bibliografici}

Acquaviva, S. e R. Stella (1989), Fine di un'ideologia: la secolarizzazione, Roma, Borla.

Arculeo, A. e A. Marradi (1985), Elezioni e referenda negli anni settanta, in «Rivista italiana di scienza politica», XV, pp. 99-141.

Berger, P. (1984), La sacra volta, Milano, SugarCo.

- (1987), L'imperativo eretico, Torino, Lcd.

Brunetta, G. e L. Longo (a cura di) (1991a), Italia cattolica, Firenze, Vallecchi.

- (1991b), Il cattolicesimo degli italiani: risultati dell'indagine, in Brunetta e Longo (1991a), pp. 19-86.

Bruschi, A. (1990), Conoscenza e metodo, Milano, Bruno Mondadori.

Calvaruso, C. e S. Abbruzzese (1985), Indagine sui valori in Italia. Dai postmaterialismi alla ricerca di senso, Torino, Sei.

Cappello, F.S. (1992), Piume nel soffio del signore: donne e religione di chiesa, in S. Burgalassi e S. Martelli (a cura di), Immagini della religiosità in Italia, Milano, Angeli.

Cartocci, R. (1984), Concetti e indicatori: il contributo della nuova retorica, in Marradi (1984b), pp. 69-98.

- (1990), Elettori in Italia. Riflessioni sulle vicende elettorali degli anni ottanta, Bologna, il Mulino.

- (1991a), Scambio, appartenenza, integrazione: la risposta locale, in «il Mulino», XL, pp. 721-732.

- (1991b), Localismo e protesta politica, in «Rivista italiana di scienza politica», XXI, pp. 551-581.

- (1993), Consenso, disgregazione sociale e ricerca di identità, in F.L. Cavazza (a cura di), La riconquista dell'Italia, Milano, Longanesi, pp. $415-434$.

Cavalli, A. e A. De Lillo (1988), Giovani anni 80, Bologna, il Mulino.

Cazeneuve, J. (1974), La sociologia del rito, Milano, Il Saggiatore.

Corbetta, P. (1992), Metodi di analisi multivariata per le scienze sociali, Bologna, il Mulino.

Crespi, F. (1988), La fine della secolarizzazione: dalla sociologia del progresso alla sociologia dell'esistenza, in «Studi di sociologia», n. 1 .

Diamanti, I. e G. Riccamboni (1992), La parabola del voto bianco, Vicenza, Pozza.

Fisichella, D. (a cura di) (1988), Metodo scientifico e ricerca politica, Roma, Nuova Italia Scientifica.

Garelli, F. (1991), Religione e chiesa in Italia, Bologna, il Mulino.

Geertz, C. (1973), Interpretation of Cultures, New York, Basic Books; trad. it. Interpretazione di culture, Bologna, il Mulino.

Giampaglia, G. (1992), Dai modelli a indicatori multipli ai modelli LISREL: una rivoluzione silenziosa nella ricerca sociale, relazione presentata alla sezione di Metodologia del convegno nazionale dell'AIS, Pisa, 28-31 ottobre 1992. 
Gubert, R. (a cura di) (1992), Persistenze e mutamenti dei valori degli italiani nel contesto europeo, Trento, Reverdito.

Guidorossi, G. (1984), Gli italiani e la politica, Milano, Angeli.

Guizzardi, G. (1979), La religione della crisi, Milano, Comunità.

- (1991), Cattolicesimo e religiosità. Alcune tendenze, in Brunetta e Longo (1991a), pp. 284-297.

Lazarsfeld, P.F. (1967), Problemi di metodologia, in P.F. Lazarsfeld, Metodologia e ricerca sociologica, Bologna, il Mulino, pp. 179-229.

LeBras, G. (1969), Studi di sociologia religiosa, Milano, Feltrinelli.

Longo, A. (1991), La pratica religiosa: fedeli, osservanti, spettatori, in Brunetta e Longo (1991a), pp. 156-170.

March, J.C. e J.P. Olsen (1992), Riscoprire le istituzioni, Bologna, il Mulino, 1992.

Margiotta-Broglio, F. (1991), I cattolici fra Stato e Chiesa, in Brunetta e Longo (1991a), pp. 309-319.

Marradi, A. (1974), Analisi del referendum sul divorzio, in «Rivista Italiana di Scienza Politica», IV, pp. 589-644.

- (1984a), Concetti e metodo nelle scienze sociali, Firenze, Giuntina.

- (a cura di) (1984b), Metascienza, Roma, La goliardica.

- (1991), La nuvola del concetto, in «Democrazia diretta», VI, n. 3, pp. 32-39.

- (1993), Referenti, pensiero e linguaggio, di prossima pubblicazione in «Sociologia e ricerca sociale», XV.

Nesti, A. (1992), Religione e cultura. Il cattolicesimo nell'Italia contemporanea, in «Religione e società», VII, pp. 37-61.

Ogden, C.K. e I.A. Richards (1946), The Meaning of Meaning, New York, Harcourt Brace Jovanovitch; trad. it. Il significato del significato, Milano, il Saggiatore, 1965.

Parisi, A. (1978), Tra ripresa ecclesiastica ed eclissi della secolarizzazio$n e$, in «Città e regione», n. 7, pp. 32-46.

- (1979), Un partito di cattolici? L'appartenenza religiosa e $i$ rapporti col mondo cattolico, in A. Parisi (a cura di), Democristiani, pp. 85-152.

Perelman, C. (1981), Il dominio retorico, Torino, Einaudi.

Perelman, C. e L. Olbrechts-Tyteca (1966), Trattato dell'argomentazione, Torino, Einaudi.

Peri, P. (1988), La stampa periodica settimanale in Italia: diffusione $e$ pubblico, in «Polis», II, pp. 533-554.

Ricolfi, L. (1988), Il processo di secolarizzazione in Italia nel dopoguer$r a$, in «Rassegna italiana di sociologia», XXIX, pp. 37-87.

- (1992), Sul rapporto di indicazione: l'interpretazione semantica $e$ l'interpretazione sintattica, dattiloscritto presentato al seminario della sezione di Metodologia dell'AIS, Pavia, 29 maggio 1992.

Rositi, F. (1992), Strutture di senso e strutture di dati, dattiloscritto presentato al seminario della sezione di Metodologia dell'AIS, $\mathrm{Pa}$ via, 29 maggio 1992. 
Sani, G. (1991), Church Attendance and the Vote for the DC: Evidence from the 1980s, in «Italian Politics and Society News letter», n. 34, pp. 13-18.

- (1993), Le Italie del 5 aprile, di prossima pubblicazione in «Polis», VII.

Sartori, G. (1984), Guidelines for Concept Analysis, in G. Sartori (a cura di), Social Science Concepts, London, Sage, pp. 15-85.

Sartori, L. (1991), La pratica religiosa: omologazioni e differenze, in Brunetta e Longo (1991a), pp. 171-192.

Scartezzini, R. (1992), Valori religiosi e contesti socio-culturali, in $\mathrm{Gu}$ bert (1992), pp. 451-497.

Schadee, H.M.A. (1990), Lavorare sui dati: dalle cattedrali nel deserto agli artigiani telematici, in «Cattaneo», VII, Bologna.

Toulmin, S. (1975), Gli usi dell'argomentazione, Torino, Rosemberg e Sellier.

Tullio-Altan, C. (1974), I valori difficili, Milano, Bompiani.

- (1986), La nostra Italia, Milano, Feltrinelli.

- (1989), Populismo e trasformismo. Saggio sulle ideologie politiche italiane, Milano, Feltrinelli.

- (1992), Soggetto, simbolo e valore. Per un'ermeneutica antropologica, Milano, Feltrinelli.

Turner, V. (1986), Dal rito al teatro, Bologna, il Mulino.

Van Gennep, A. (1909), Les rites de passage, Paris, Nourry; trad. it. I riti di passaggio, Torino, Boringhieri, 1981.

Weber, M. (1973), Il lavoro intellettuale come professione, Torino, Einaudi.

Wilson, B.R. (1986), La religione nel mondo contemporaneo, Bologna, il Mulino. 